\title{
Telemedicine in Complex Diabetes Management
}

\author{
Marie E. McDonnell ${ }^{1}$ \\ Published online: 24 May 2018 \\ (C) Springer Science+Business Media, LLC, part of Springer Nature 2018
}

\begin{abstract}
Purpose of Review Telehealth has the potential to positively transform the quality and cost-effectiveness of complex diabetes management in adults. This review explores the landscape of telemedicine approaches and evidence for incorporation into general practice.

Recent Findings Telemedicine for diabetes care is feasible based on over 100 randomized clinical trials. Evidence shows modest benefits in A1c lowering and other clinical outcomes that are better sustained over time vs. usual care. While telemedicine interventions are likely cost-effective in diabetes care, more research is needed using implementation science approaches.

Summary Telehealth platforms have been shown to be both feasible and effective for health care delivery in diabetes, although there are many caveats that require tailoring to the institution, clinician, and patient population. Research in diabetes telehealth should focus next on how to increase access to patients who are known to be marginalized from traditional models of health care.
\end{abstract}

Keywords Diabetes $\cdot$ Telemedicine $\cdot$ Virtual visits $\cdot$ Type 1 diabetes $\cdot$ Type 2 diabetes $\cdot$ Complex diabetes $\cdot$ Virtual care

\section{Introduction}

In 2013, a large analysis of claims based data showed that one out of every five health care dollars was spent on diabetesrelated care, with the total cost adding up to approximately eight times the National Institutes of Health's budget for all biomedical research [1]. The current cost of diabetes care now is nearing $\$ 300$ billion per year, over a $40 \%$ increase from the last decade, and representing about $12 \%$ of USA's total health care expenditures $[1,2]$. However, despite the enormous investment in health care, Americans overall report being unsatisfied with health care compared with most other developed (and some developing) countries [3]. This appears likely to be due to a perceived lack of access to the desired care at the time needed. In the case of a chronic disease epidemic such as diabetes, access to evidence-based effective care has become

This article is part of the Topical Collection on Health Care Delivery Systems and Implementation in Diabetes

Marie E. McDonnell

MMCDONNELL@BWH.HARVARD.EDU

1 Division of Endocrinology Diabetes and Hypertension, Brigham and Women's Hospital, 221 Longwood Avenue, Suite 381, Boston, MA 02115, USA increasingly difficult for the health care system; yet, at the same time, we have accumulated more evidence that diabetes care models are generally effective. Diabetes-related complication rates have declined [4] and traditional models of care appear especially effective when they utilize team-based innovations [5]. However, the challenge recognized by leaders in the field is that even though the risk of complications is decreasing in people with established diabetes, the overall prevalence of complications has stabilized due to the consistent rise in prevalence since the late 1980s [6]. Over the past decade, it has become clear that reduction of diabetes disease burden will require more reliable access to evidence-based quality care. These effective care models were obviously tested in populations with clear access to classical medical care structures [7, 8]. Novel approaches, such as electronic solutions, designed to increase access to this care are now necessary.

Telehealth is the use of a technology-based virtual platform to deliver various aspects of health information, prevention, monitoring, and medical care. Telehealth's largest segment is telemedicine, which is generally defined as the practice of medicine via a remote electronic interface. This approach to care delivery has been developed since the early 1990s to increase convenience and overall access to care. For the purposes of this review, there are three types of telemedicinebased care delivery: clinician to patient, clinician to clinician, 
and patient to surrogate (coach, pharmacy technician, patient navigator, or interactive module/game). Telemedicine services can also be categorized in chronological terms: real-time virtual consultation (synchronous); stepwise where data is uploaded for review by a clinician prior to a consult (asynchronous); and remote patient monitoring (continuous). In diabetes care, all of these types of telemedicine delivery forms have been used and studied to some extent.

The focus of this review is on specialty-driven, synchronous telehealth innovations designed to deliver complex disease management to relatively small populations of adult patients with diabetes. This is in clear contrast to a health systems population approach to care that might grant large populations access to general care through synchronous telemedicine services. In fact, evidence to support the adoption of system-wide direct-to-consumer videoconferencing interventions, such as those offered by such companies, is lacking. In a recent study using claims data from 300,000 patients with respiratory conditions, investigators found that $12 \%$ of telehealth visits replaced traditional visits to other providers and $88 \%$ represented new utilization. The authors concluded that Teladoc(C-type services do increase access to care but appeared to increase utilization and health care spending overall [9]. Importantly, this study addressed the payer perspective alone and does not consider potential benefits of telehealth solutions for special patient populations whose diminished access could increase overall cost of care.

This review will not include the following areas which have included recent innovations in telehealth: prevention [10]; inpatient care [11]; pediatric and adolescent diabetes. Also not discussed are details on eHealth, or electronic health solutions which generally include smartphone-based bioanalytic and diagnostic applications. Such technologies will be described only in the context of integration with cointerventions designed for diabetes care.

\section{Telemedicine Providers and Program Design}

Given that the team concept is a deeply ingrained element of effective diabetes care, telemedicine in diabetes lends itself well to incorporating clinicians beyond the physician.

Just as in existing care models in the USA and beyond, interim support via formal telecommunication can effectively be given by non-providers such as medical assistants, licensed practice nurses, or registered nurses. With a dramatic increase in smartphone use in the last decade, more patients have the required technology at their fingertips, creating the opportunity for population-based intensive diabetes management. For example, the pairing of telehealth with biometrics (e.g., remote blood glucose monitoring) and bioanalytics (e.g., average steps per week from an activity tracking device) is a common and often important strategy for comprehensive chronic disease management. In diabetes, the best example is remote glucose monitoring systems that utilize smartphone platforms for Bluetooth and cloud-based data storage, which allows for sharing data between patients and providers. This allows for glucose monitoring of a population, or patient panel, by nonproviders using algorithms to identify individuals who need real-time input or acute intervention (e.g., for recurrent hypoor hyperglycemia). Such systems, which have been standard practice for insulin pump and continuous glucose monitoring systems, also allow for real-time data sharing during virtual consultation, or virtual visits (VV). Most electronic health record systems can integrate patient-collected glucose (and blood pressure, weight, etc.) data into the standard platform. One could envision a model where patients are provided with high-intensity, data-driven, and personal interim care by nonphysicians at a lower cost, thus limiting physician visits to once or twice yearly and/or for urgent evaluations [12]. This type of high-touch yet virtual approach to diabetes management could be bolstered by online training and education using low-cost audiovisual designs and platforms that can be administered by members of the patient's care team [13].

Available evidence does emphasize that in complex diabetes, sharing of biometric data requires a high level of patient engagement (and sometimes patience). Moreover, sharing of self-measured data electronically is not effective on its own when compared with traditional methodologies such as keeping a paper logbook and bringing it to scheduled appointments. A good example from the literature is the pregnant patient who is managing pre-gestational diabetes. A Cochrane systematic review addressed techniques of monitoring blood glucose during pregnancy for women with type 1 or type 2 diabetes. Albeit with little high quality data, the review found no evidence that any glucose monitoring technique is superior to any other technique in this typically highly engaged community of patients [14]. Future research on the utility of remote biometric self-monitoring needs to address two key unresolved questions: (1) how to improve care for less engaged patient populations and (2) how the integration of biometrics into telemedicine programs can optimize outcomes and cost-effectiveness in the care of patients who are already engaged.

Unfortunately, financial support for non-physician interactions has been a limitation in widespread adoption of the integrated team model in telemedicine. Currently, the health system will only directly reimburse for telemedicine services delivered by licensed providers including physicians, advanced practice nurses or nurse practitioners, and physician assistants. For this reason, it has been difficult for most institutions and payers to develop a comprehensive specialty-level telemedicine service in diabetes. Other options for payment include incorporation into chronic care management (CCM) services. Through CPT code 99490, Medicare pays approximately $\$ 44$ per patient per month to providers who deliver 
$20+$ min of non-face-to-face care coordination services to eligible Medicare beneficiaries. Other insurers have followed and in 2017, a new complex CCM codes were added along with more reimbursement.

Also important to consider, while the at-home "do it yourself" nature of telehealth is very attractive to patients especially as they require more medical interventions as they age, evidence to show clear benefit of self-management of diabetes using telehealth platforms is limited. Similar to hypertension, there is both a lack of evidence and an inherent need to patient guidance due to the relatively complex risk/benefit assessment required for most management approaches [15]. As a result, most studies have involved a high amount of clinician time in the interventions.

\section{Best Evidence for Telehealth in Diabetes Populations}

\section{Clinician to Patient}

The use of telemedicine in the care of patients with diabetes has been studied for two decades in diabetes and has been repeatedly demonstrated to be feasible. The effectiveness of the interventions has been unclear, however. In a recent metaanalysis, the authors included 111 randomized clinical trials using synchronous telemedicine approaches in diabetes patients and chose change in A1c as the clinical outcome. Compared with usual care, the authors found that most studies show what they described as "modest" improvements in glycemic control. However, the overall difference in mean A1c after 3 months was $-0.57 \%(-0.74$ to -0.40$)$ and at 12 months $-0.26 \%,-0.46$ to $0.06 \%$ [16•]. This degree of change is approximately what would be expected from the addition of an effective diabetes medication that was adhered to $>50 \%$ of the time. As the participants were randomized to the telemedicine interventions, the impact was not simply about access to a new medication but rather to a new care delivery method. Lastly, telemedicine had no clear positive or negative effect on mortality, patient quality of life, or hypoglycemic episodes.

Interestingly, other studies and meta-analyses have similarly identified small but clinically significant benefits and considered them to be underwhelming. The combination of unrealistic expectations and patient selection has led to lackluster results in several studies and may have resulted in a delay in both buy-in and broad implementation in diabetes populations. An early example of this is the Informatics for Diabetes Education and Telemedicine (IDEATel) study published in 2009 [17••]. The IDEATel study was a large proof-of-concept trial performed in older adults on Medicare (the project's funder) in New York State. In the case of IDEATel, the A1c reduction over time was modestly lower than the usual care but it was remarkably sustained over time (Fig. 1). Of importance, the baseline A1c was already in the goal target range $(<7.5)$ currently supported by the multiple societies for older adults with diabetes [18, 19]. In this case, A1c lowering in either group would be expected to be minimal but yet was still demonstrated. Perhaps the most remarkable finding was that subjects in the intervention sustained the lowered A1c whereas the usual care group did not. This suggested that the continued engagement allowed by the program had an impact on sustaining efforts to control glucose. There were also significant benefits seen in blood pressure lowering.

Although sustaining glucose and blood pressure control pose one of the greatest challenge to diabetes care and has been shown in many studies to be difficult following intensive in-person interventions [8, 20], this finding was not a predefined outcome. More importantly, the intervention costs $\$ 8000$ per patient over 5 years, making the costeffectiveness analysis unfavorable toward the intervention for direct implementation [17••]. However, using modern technology, this cost would be reduced severalfold per person, to the cost of a Bluetooth-enabled glucose meter, laptop computer, and/or smartphone and a mobile phone account. In the final estimate of cost-effectiveness, costs incurred by the patient in the course of usual in-person interim care would have to be subtracted. Among these are travel expenses, loss of productivity due to missed work days, and technical overhead costs included in medical charges.

What was the most crucial element in IDEATel that yielded sustained improvements in A1c and BP? If this element were relatively inexpensive such as 15 min per week of telephonic interaction, then widespread adoption could yield substantial population-level disease control. Another relevant question is whether to focus on lifestyle or medication management with any tele-intervention management of a complex disease. To this end, important efforts to study stripped down approaches have been encouraging. One study randomized 202 patients with baseline A $1 \mathrm{c}>7.5 \%$ and taking at least two medications to 12 weeks of either telemedical coaching or usual care. Both groups received step counters and weighing scales but the Telemedical Lifestyle Intervention Program (TeLiPro) group also received medical-mental motivation, a formula diet, and self-monitored blood glucose for 12 weeks. The A1c reduction was significantly higher in the TeLiPro group (mean $\pm \mathrm{SD}-1.1 \pm 1.2$ vs. $-0.2 \pm 0.8 \%$; $P<0.0001$ ), which was sustained after adjusting for baseline variables and after 52 weeks following the program's end. Although the actual intervention was likely the enhanced therapeutic strategy rather than telemedicine itself, it demonstrated that complex lifestyle intervention can be administered remotely and at a lower cost [21]. 
Fig. 1 Results from the IDEATel study. (Republished with permission of $[17 \cdot \bullet])$
A

Hemoglobin A1c (\%)

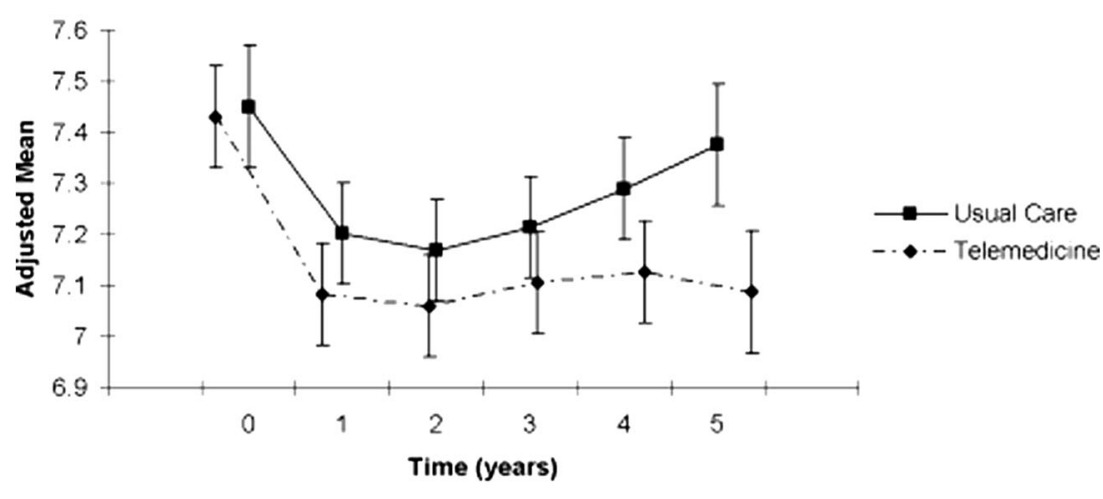

B

LDL Cholesterol (mg/dL)

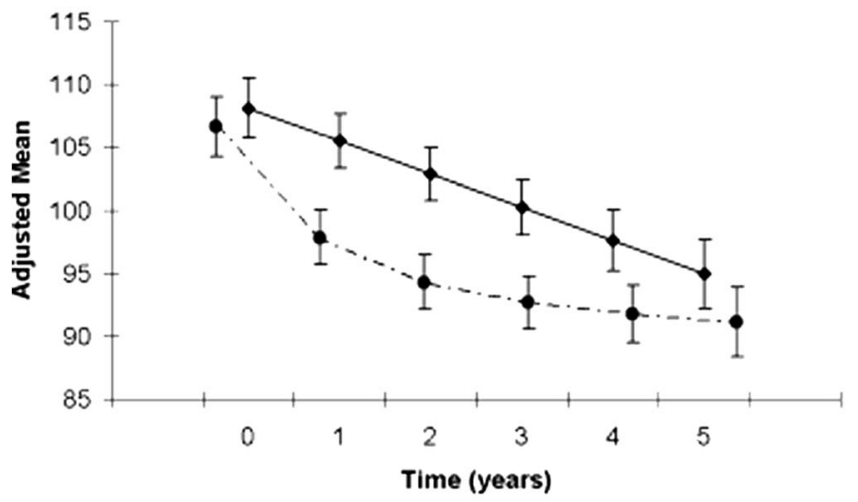

C

Blood Pressure $(\mathrm{mmHg})$

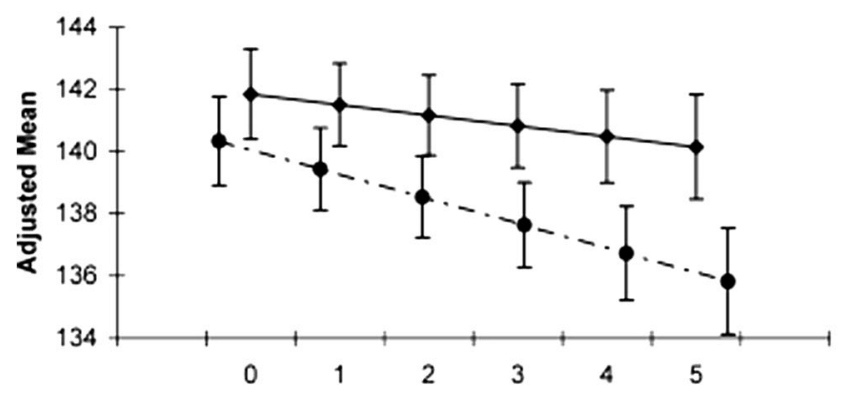

Systolic

$\longrightarrow$ Usual Care

- - Telemedicine

\section{Clinician to Clinician}

Telementoring is a viable option for population management of complex diabetes whereby diabetes specialists provide case-based guidance and education on regimen intensification to non-specialist clinicians. Designed to optimize care in remote, underserved areas, Project Echo is a foundational telementoring program launched in New Mexico. The Project Echo model engages specialists in one location with clinicians in another via teleconference, which may include specific case discussions and/or didactic lectures. A derivative of Project Echo, the "Endo Echo" model, is a diabetes carefocused telementoring program that is being implemented and studied at several sites [22].

\section{Patient to Surrogate}

Approaches designed to impact larger populations of patients primarily with type 2 diabetes are of major interest given the epidemic-level prevalence of the disease. Few, however, have been adequately tested. Telehealth approaches such as coaching have been evaluated and in some cases formally investigated as effective methods to augment usual care. One example is online team-based gaming.

Researchers from the veterans administration (VA) developed an online, team-based game designed to teach patients about diabetes self-management and tested its impact on glycemic control in participants with type 2 diabetes. The study enrolled 456 Veteran Affairs patients from the eastern USA, 
recruiting participants with diabetes who had inadequate glucose control while taking oral diabetes medications. Subjects who were randomly assigned to play the diabetes selfmanagement education game had significantly greater reductions in A1c over 6 months than controls assigned to a civics education game. It is important to note the high level of patient engagement with approximately $89 \%$ of participants requested to participate in future programs using this game. A1c levels were tested at enrollment, 6 months and 12 months after the launch of the game. Overall, diabetes game participants had significant reductions in A1c levels (a drop of $0.74 \%$ compared to $0.44 \%$ for the control group) and as is typically seen with effective therapeutic strategies those with higher A1c had greater reductions.

The study was not designed to assess which aspect of the educational game led the outcome, such as the content of the game, the community or competition-based nature of the game; or some combination of factors [23]. Several studies are ongoing to address adherence and the potential impact of increased adherence, in different diabetes populations [24, 25].

\section{Telehealth for Special Diabetes Populations}

There are certain conditions that lend themselves extremely well to telehealth. Perhaps the clearest example is "telestroke" programs that are now available in most parts of the world, which allow for real-time telementoring to weigh the risk and benefit of thrombolytic therapy during the acute evaluation of stroke victims. In diabetes, ophthalmology led the way in telehealth via remote, nonmydriatic retinal screening programs. For example, one large-scale telemedicine diabetic retinopathy (DR) screening program screened 21,222 patients in 2 years increased screening rates by $16 \%$, dramatically reduced the need for at-risk patients to consult with an eye care specialist, and reduced wait times in one of the largest safetynet health care systems in Los Angeles, CA [26].

In contrast, telemedicine for chronic management of special diabetes populations has focused mostly on type 1 diabetes. In a 2017 metanalysis, the authors selected 38 studies that met their criteria, which included RCTs, type 1 diabetes population only, and any form of clearly defined telemedicine according to the American Telemedicine Association. These interventions could include tele-education, telemonitoring, teleconsultation, telecase management, or telementoring. Interventions could include a variety of methodologies and platforms: fax, short message system, internet-based communication, telephone, mobile phone. The outcome had to be to improve clinical health status. Among the 38 studies included, few studies focused on synchronous telemedicine approaches such as VVs $(N=5)$ and the majority $(N=19)$ were simple and involved transmission of blood glucose data with feedback. Overall, the A1c reduction was modest, $0.18 \%$, which was heavily weighted by two studies, one of which studied synchronous VVs with patients along with data sharing. The analysis also included both adult and children, and in a subgroup analysis, they found that adults appear to benefit more than adolescents, children, or pregnant women at the end of the study. Also of interest was that the telemedicine effectiveness was higher in studies that lasted at least 6 months, suggesting the need for adjustment time to new approaches. In the end, only tele-education and VVs had a positive effect on glycemic control and overall, the effect was small. What was not measured in this metanalysis is a change in patient satisfaction or access to care. The authors also noted the lack of reporting on other outcomes such as ethnicity or socioeconomic status $[27 \bullet]$.

In the author's experience, special populations are especially amenable to telemedicine approaches, especially if their condition requires both special expertise and frequent access to clinical input. Two examples include the pregnant woman with diabetes and the patient with cystic fibrosis-related diabetes. In the first case, frequent, brief, and data-driven touch points define the approach to diabetes care, which is well suited to virtual visitation paired with glucose data sharing. In the second case, this CFRD patient risks acquiring an infection with each visit to health care facilities and already has several medical visits per year for CF Care; virtual visitation poses special benefits outside of glycemic control in this population. Other examples of high-benefit patient populations include the adult with disabilities, patients new to technology, or insulin and follow-up after discharge from the hospital.

\section{Institutional Experience}

In March of 2015, Brigham and Women's Hospital (BWH) in Boston launched an institutionally supported telemedicine program with VVs being the flagship service offered. Providers were encouraged to adopt this visit type and incorporate it into their usual practices. In order to support this program, the hospital funded physicians and nurse practitioners directly, paying them a fee for each 15 or $30 \mathrm{~min}$ visit. Within the first year, Endocrinology quickly became the leading clinical telemedicine program in the hospital, with most VVs being conducted between pregnant women and their physicians for diabetes management. Now, in the fourth year of this program, approximately $50 \%$ of the $\mathrm{VVs}$ being done currently are with pregnant women with diabetes with the remainder including patients with complex diabetes and other endocrine conditions. Most patients who choose VV do so because they require frequent contact with their clinical team (e.g., for insulin adjustments) and they feel comfortable with electronic communication. Among 251 respondents to a survey offered to all VV patients at Brigham and Women's Hospital, 
95\% of patients reported that they satisfied with their virtual visit and $94 \%$ of patients would recommend a virtual visit to another patient with similar health problems. Importantly, 95\% of patient said that it was easier to access their provider with a virtual visit and $70 \%$ of patients said they were able to be seen sooner for their follow up appointment. Lastly, and most critical to the question of relevance of this service in the marketplace of care options available to patients, $88 \%$ of patients felt that a virtual visit addressed their needs to the same degree of an inperson visits for their particular problem.

However, as a result of this investment, we and other hospital systems have learned there are many pitfalls and challenges to adopting telemedicine practices into usual care delivery. We continue to learn from the experience of other hospitals and systems. In a blog post, Emme Deland and her colleagues at New York Presbyterian [28] presented a wellorganized cache of challenges that presents itself early on in the adoption of a telehealth program, which was launched July, 2016. Their challenges are shared by other programs and include liability, licensure, reimbursement, and workflow. From a liability perspective, the question that is asked is if the telehealth approach, for example virtual visits, creates new medical practice liabilities. As the authors discovered, most malpractice carriers cover telehealth practices as long as the individual clinician practices within his/her license. The problem of licensure can impact a program that intends to serve patients across states lines to optimize access and quality of a program. At this time, state medical licensure regulations unfortunately do not anticipate the novel scenarios created by telehealth. For this reason, in most cases, clinicians cannot have a visit with a patient in a state in which the clinician is not licensed, although this is variable according to the American Telemedicine Association's state-by-state guide [29]. One solution is to identify the states most commonly represented in the pool of patients targeted for the telehealth program and obtain licenses for the clinicians in each (New York Presbyterian (NYP) reported licensing urgent care physicians in four states). Although change in this regard has been slow, there are ongoing efforts to recognize the importance of interstate licensure, which would reduce a significant barrier to telehealth programs designed to reach unique patient populations. One example of a positive step in this direction is the Interstate Medical Licensure Compact (IMLC), which expedites physician licensing across its 22 member states through a voluntary expedited pathway for those who wish to practice in multiple states. As illustrated on the website, the IMLC is working to expand membership across the USA in order to increase access to telemedicine technologies. Reimbursement is a key to sustainability of any health delivery service especially in a largely fee-for-service environment. Most insurance companies do not reimburse clinicians or hospitals for virtual visits and when they do, there are often limitations. Medicare, for example, only reimburses a certain set of telehealth services for patients located in designated rural physician shortage areas. As was done at NYP, several hospital systems, including Brigham and Women's Hospital, had to implement a transition strategy with the hope that reimbursement policies of payers would change, especially if early outcomes are demonstrably positive. There are generally two options for transitional reimbursement methods. One is to offer telehealth as a premium cash-pay service, which keeps the program solvent but limits access and poses the risk of enrolling too few patients to demonstrate early evidence of benefit to payers. The other is for the hospital to make a short-term but substantial investment by offering the service for free (or with copayment from reimbursing payers) and compensate its clinicians directly for their time. This poses a financial risk but increases volume relatively quickly, allowing for more robust outcomes earlier in the implementation. Beyond reimbursement, in diabetes as with other chronic diseases, the virtual visit has to "count" as a valid medical encounter. Medicare and some other insurances require that patients who are on insulin pump therapy must have a documented patient visit every 3 months or they risk not having their supplies shipped to them and/or covered. Despite the convenience of the virtual visit for these patients and the availability of virtually viewable glucose data, telehealth visits currently do not fill this requirement.

Finally, workflow is the rate-limiting step for any individual provider, clinic, or hospital system that must be addressed head on before one can expect any degree of success from a telehealth program. The first workflow item that systems and providers alike need to develop is identifying adequate and available space. Although the footprint of a VV office can be quite small and efficient for busy offices, it needs to be private to accommodate conversations with patients. Practices are creating small virtual offices and also encourage providers to use their administrative offices for these VVs as well.

Scheduling virtual visits requires careful planning and thought. Virtual visits should be scheduled using the same system as in-person visits. Most EHRs are able to accommodate unique visit types and templates for these visits, allowing both the clinician and the administrative staff to easily distinguish virtual visits from usual visits in the clinic schedule interface. In our institution, virtual visits are scheduled for $30 \mathrm{~min}$ for high complexity visits and $15 \mathrm{~min}$ for low complexity; however, as billing is now streamlined to allow for use of a modifier "on top of" usual billing, VVs can be booked similarly to traditional visits. Visit documentation should also be the same other than a limited physical examination. A benefit to $\mathrm{VV}$ is that the usual clinic rooming procedure, with its associated delays, is not a factor. For this reason, VVs are more amenable to timely service for the patient. However, blending VVs into a usual clinic session often leads to delays, with patients waiting in "virtual waiting rooms" for their clinician to connect. Therefore, scheduling VVs at the beginning of a session, or having sessions dedicated to VV only, are the 
most effective approaches to incorporation of $\mathrm{VV}$ into routine clinic schedules. The second critical workflow item is information technology (IT) support. IT supports both before and in real-time during visits is a critical element of any successful telemedicine program. Dedicated IT staff contact patients prior to their first VV in order to education patients on how to use the software, their own hardware for best results, and to ensure adequate connectivity in the location they plan to be for their visit. The third critical item in the workflow is training of office staff in proper booking of VVs, which includes using the appropriate scheduling templates and connecting the IT support team with the patient for pre-visit preparation.

\section{Conclusions and Next Steps}

Telemedicine platforms have been shown to be both feasible and effective for health care delivery in diabetes, although there are many caveats that require careful tailoring to the institution, clinician, and patient population in order for virtual care to make an institutional impact (Table 1). While telemedicine interventions appear mostly effective, and perhaps cost-effective, when applied to special populations, there are cautions to be heeded from recent literature. For example, despite a global enthusiasm for virtual health care, virtually administered interventions represent a sea change for many patient populations. In studies showing minimal or negative results, most identify fundamental limitations in trial populations such as lack of internet access, which may be related to education level, age, or other unmeasured factors [30]. Most of what we understand about how telehealth practices fare in real-world health care institutions is still at the anecdotal level. System readiness tools have not been adequately developed, tested, nor promulgated [31]. Cost-effectiveness is perhaps the most important outcome of telehealth interventions since this, when done correctly, combines quality (clinical success) and investment (both from the clinicians, institution, and health care payers). In terms of calculating costeffectiveness specifically for telehealth, one has to also consider the different elements involved in a telehealth program and the relative value of each. As we have learned from prior studies, with the exception of physical activity e-health platforms [32], biometric data collection alone is costly and ineffective, while the combination of biometrics and co-interventions can produce powerful clinical outcomes. This has been best demonstrated in programs focused on remote blood pressure monitoring and medication titration [33] and ought to be transferable to remote glucose monitoring. However, as we learned from the IDEATel study, the selection of the patient population (e.g., low vs. high baseline disease severity), the initial investment (userowned technology vs. custom designed), and the prespecified outcome (e.g., lowering of A1c vs. long-term maintenance of initial lowering) are critical pieces that must be considered when envisioning the cost-effectiveness of any telemedicine program in diabetes. We need more pragmatic trials and natural experiments in telehealth that provide enough variety along the dimensions of time, geography, and content in order to yield useful practice standards that health systems and patients can employ for optimal results [34].

In the next decade, research in the area of telehealth in diabetes should focus on how to leverage technology to increase access to marginalized patients. These are patients who are not already engaged in their health or the health system that is available to them. As a result, marginalized patients present for care at more advanced, and costly, stages; in

Table 1 Telemedicine in complex diabetes care: ingredients for impact*

\begin{tabular}{|c|c|c|c|c|c|}
\hline Menu of services & $\begin{array}{l}\text { Institutional } \\
\text { culture }\end{array}$ & Biometric data sharing & Technical support & Patient access & Financial feasibility \\
\hline $\begin{array}{l}\text { Virtual visits } \\
\text { Remote biometric data } \\
\text { monitoring (e.g., glucose, } \\
\text { data downloaded from } \\
\text { insulin pumps, etc) } \\
\text { Secure electronic messaging } \\
\text { between clinician and } \\
\text { patient } \\
\text { Coaching: telephonic/texting } \\
\text { vs. game based } \\
\text { Telementoring program }\end{array}$ & $\begin{array}{l}\text { Provider } \\
\text { engagement } \\
\text { Provider } \\
\text { champions } \\
\text { Select } \\
\text { high-yield } \\
\text { engaged } \\
\text { populations }\end{array}$ & $\begin{array}{l}\text { Electronic data-sharing for } \\
\text { glucose, blood pressure, } \\
\text { and/or body weight } \\
\text { Patient-collected data } \\
\text { received by EHR platform }\end{array}$ & $\begin{array}{l}\text { Central and on-site } \\
\text { information } \\
\text { technology support } \\
\text { staff }\end{array}$ & $\begin{array}{l}\text { Targeting } \\
\text { populations } \\
\text { most likely to } \\
\text { benefit* } \\
\text { Multi-state access }\end{array}$ & $\begin{array}{l}\text { Payment structure to } \\
\text { incentivize clinicians, } \\
\text { institutions, and patients }\end{array}$ \\
\hline
\end{tabular}

*Common elements of successful comprehensive diabetes telemedicine programs. Domains include services provided, institutional culture, data sharing availability, technical support, patient access, and financial feasibility

Examples: diabetes in pregnancy; cystic fibrosis related diabetes; individuals with disabilities/travel limitations; new to technology or insulin; follow-up after discharge from the hospital 
diabetes, this problem is the underpinning of the exorbitant and unsustainable costs of inpatient care. At BWH, when we evaluated the impact of our program on access, the vast majority of patients reported that they would have come to an inperson visit if the VV was unavailable. It is clear that telemedicine is generally offered to (and studied in) patients who are already engaged in the health system. Given this natural tendency of telemedicine, which is to engage the already engaged, the idea may be for telemedicine to serve as an alternative lower cost service for stable patients so that in-person costly care can be reserved for new diagnoses, disease exacerbations, hands-on device education, and the like. In this way, costs can be lowered overall while not sacrificing, and potentially increasing, quality of care.

On a final note, a discussion about population level strategies for diabetes management must also address the problem of prevention. Telehealth strategies for prevention have also been shown to be effective and outcomes of large-scale implementation will hopefully lead the way toward global strategies to combat the diabetes pandemic.

\section{Compliance with Ethical Standards}

Conflict of Interest Marie E. McDonnell declares that she has no conflict of interest.

Human and Animal Rights and Informed Consent This article does not contain any studies with human or animal subjects performed by the author.

\section{References}

Papers of particular interest, published recently, have been

highlighted as:

- Of importance

•• Of major importance

1. American Diabetes, A. Economic costs of diabetes in the U.S. in 2012. Diabetes Care. 2013;36(4):1033-46.

2. Health Expenditure, total \% of GDP. 2017 1/1/2018; Available from: https://data.worldbank.org/indicator/SH.XPD.TOTL.ZS? locations $=\mathrm{US}$.

3. Hero JO, Blendon RJ, Zaslavsky AM, Campbell AL. Understanding what makes Americans dissatisfied with their health care system: an international comparison. Health Aff (Millwood). 2016;35(3):502-9.

4. Gregg EW, Williams DE, Geiss L. Changes in diabetes-related complications in the United States. N Engl J Med. 2014;371(3): 286-7.

5. Shojania KG, Ranji SR, McDonald KM, Grimshaw JM, Sundaram $\mathrm{V}$, Rushakoff RJ, et al. Effects of quality improvement strategies for type 2 diabetes on glycemic control: a meta-regression analysis. JAMA. 2006;296(4):427-40.

6. Seaquist ER. Addressing the burden of diabetes. JAMA. 2014;311(22):2267-8.
7. UK Prospective Diabetes Study 6. Complications in newly diagnosed type 2 diabetic patients and their association with different clinical and biochemical risk factors. Diabetes Res. 1990;13(1):111.

8. DCCT. The effect of intensive treatment of diabetes on the development and progression of long-term complications in insulindependent diabetes mellitus. The diabetes control and complications trial research group. N Engl J Med. 1993;329(14):977-86.

9. Ashwood JS, Mehrotra A, Cowling D, Uscher-Pines L. Direct-toconsumer telehealth may increase access to care but does not decrease spending. Health Aff (Millwood). 2017;36(3):485-91.

10. Sepah SC, Jiang L, Peters AL. Long-term outcomes of a Web-based diabetes prevention program: 2-year results of a single-arm longitudinal study. J Med Internet Res. 2015;17(4):e92.

11. Rushakoff RJ, Sullivan MM, MacMaster HW, Shah AD, Rajkomar A, Glidden DV, et al. Association between a virtual glucose management service and glycemic control in hospitalized adult patients: an observational study. Ann Intern Med. 2017;166(9):621-7.

12. Duffy S, Lee TH. In-person health care as option B. N Engl J Med. 2018;378(2):104-6.

13. Vidscrip. A series of videos about a health topic created by your very own healthcare provider [cited 2018 1/1/2018]; Available from: https://www.vidscrip.com/.

14. Moy FM, et al. Techniques of monitoring blood glucose during pregnancy for women with pre-existing diabetes. Cochrane Database Syst Rev. 2017;6:CD009613.

15. Mileski M, Kruse CS, Catalani J, Haderer T. Adopting telemedicine for the self-management of hypertension: systematic review. JMIR Med Inform. 2017;5(4):e41.

16. Faruque LI, et al. Effect of telemedicine on glycated hemoglobin in diabetes: a systematic review and meta-analysis of randomized trials. CMAJ. 2017;189(9):E341-64. In this meta-analysis of 111 trials, telemedicine interventions in diabetes were found overall to result in clinically significant A1c reductions.

17. Shea S, et al. A randomized trial comparing telemedicine case management with usual care in older, ethnically diverse, medically underserved patients with diabetes mellitus: 5 year results of the IDEATel study. J Am Med Inform Assoc. 2009;16(4):446-56. This is the first large randomized trial comparing synchronous virtual visits and remote data monitoring (glucose and blood pressure) to usual care in patients with diabetes. The trial demonstrated that the telemedicine approach improved A1c and blood pressure, and patients in the active group were overall more able to sustain improvement.

18. Kirkman MS, Briscoe VJ, Clark N, Florez H, Haas LB, Halter JB, et al. Diabetes in older adults: a consensus report. J Am Geriatr Soc. 2012;60(12):2342-56.

19. Garber AJ, Abrahamson MJ, Barzilay JI, Blonde L, Bloomgarden ZT, Bush MA, et al. Consensus statement by the American Association of Clinical Endocrinologists and American College of endocrinology on the comprehensive type 2 diabetes management algorithm -2017 executive summary. Endocr Pract. 2017;23(2): 207-38.

20. UKPDS. Intensive blood-glucose control with sulphonylureas or insulin compared with conventional treatment and risk of complications in patients with type 2 diabetes (UKPDS 33). UK Prospective Diabetes Study (UKPDS) Group. Lancet. 1998;352(9131):837-53.

21. Kempf K, Altpeter B, Berger J, Reuß O, Fuchs M, Schneider M, et al. Efficacy of the telemedical lifestyle intervention program TeLiPro in advanced stages of type 2 diabetes: a randomized controlled trial. Diabetes Care. 2017;40(7):863-71.

22. Bouchonville MF, Paul MM, Billings J, Kirk JB, Arora S. Taking telemedicine to the next level in diabetes population management: a review of the Endo ECHO model. Curr Diab Rep. 2016;16(10):96. 
23. Kerfoot BP, Gagnon DR, McMahon GT, Orlander JD, Kurgansky $\mathrm{KE}$, Conlin PR. A team-based online game improves blood glucose control in veterans with type 2 diabetes: a randomized controlled trial. Diabetes Care. 2017;40(9):1218-25.

24. Lauffenburger JC, Lewey J, Jan S, Nanchanatt G, Makanji S, Ferro CA, et al. Rationale and design of the ENhancing outcomes through goal assessment and generating engagement in diabetes mellitus (ENGAGE-DM) pragmatic trial. Contemp Clin Trials. 2017;59:57-63.

25. Lewey J, Wei W, Lauffenburger JC, Makanji S, Chant A, DiGeronimo J, et al. Targeted adherence intervention to reach glycemic control with insulin therapy for patients with diabetes (TARGIT-diabetes): rationale and design of a pragmatic randomised clinical trial. BMJ Open. 2017;7(10):e016551.

26. Daskivich LP, Vasquez C, Martinez C Jr, Tseng CH, Mangione CM. Implementation and evaluation of a large-scale Teleretinal diabetic retinopathy screening program in the Los Angeles County Department of Health Services. JAMA Intern Med. 2017;177(5): 642-9.

27. Lee SWH, Ooi L, Lai YK. Telemedicine for the management of glycemic control and clinical outcomes of type 1 diabetes mellitus: a systematic review and meta-analysis of randomized controlled studies. Front Pharmacol. 2017;8:330. This meta-analysis included 38 studies that investigated several telemedicine techniques in type 1 diabetes and found that adults are more likely to benefit than children.

28. NEJM Catalyst. Website 2017. Available from: https://catalyst. nejm.org/challenges-launching-telehealth-program/. Accessed 2 February 2018.

29. The American Telemedicine Association policy resource center. Website 2018. Available from: www.americantelemed.org/policypage/state-policy-resource-center. Accessed 4 April 2018

30. Kakkar A, Jacobson BC. Failure of an Internet-based health care intervention for colonoscopy preparation: a caveat for investigators. JAMA Intern Med. 2013;173(14):1374-6.

31. Yusif S, Hafeez-Baig A, Soar J. e-Health readiness assessment factors and measuring tools: a systematic review. Int J Med Inform. 2017;107:56-64.

32. McIntosh JRD, Jay S, Hadden N, Whittaker PJ. Do E-health interventions improve physical activity in young people: a systematic review. Public Health. 2017;148:140-8.

33. Tucker KL, et al. Self-monitoring of blood pressure in hypertension: a systematic review and individual patient data meta-analysis. PLoS Med. 2017;14(9):e1002389.

34. Hunter CM, McKinnon RA, Esposito L. News from the NIH: research to evaluate "natural experiments" related to obesity and diabetes. Transl Behav Med. 2014;4(2):127-9. 